Article

\title{
Fingerprint Positioning Method for Dual-Band Wi-Fi Based on Gaussian Process Regression and K-Nearest Neighbor
}

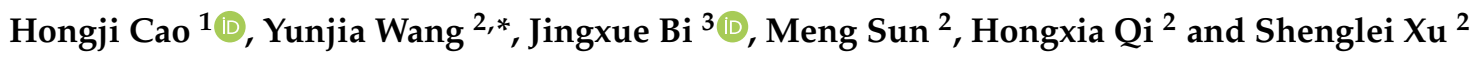 \\ 1 Key Laboratory of Land Environment and Disaster Monitoring, MNR, China University of Mining and \\ Technology, Xuzhou 221116, China; hjcao@cumt.edu.cn \\ 2 School of Environmental Science and Spatial Informatics, China University of Mining and Technology, \\ Xuzhou 221116, China; msun@cumt.edu.cn (M.S.); hongxiaqi222@cumt.edu.cn (H.Q.); \\ TB17160013B2@cumt.edu.cn (S.X.) \\ 3 College of Surveying and Geo-Informatics, Shandong Jianzhu University, Jinan 250101, China; \\ bijingxue19@sdjzu.edu.cn \\ * Correspondence: wyj4139@cumt.edu.cn; Tel.: +86-132-252-31855
}

\section{check for} updates

Citation: Cao, H.; Wang, Y.; Bi, J.; Sun, M.; Qi, H.; Xu, S. Fingerprint Positioning Method for Dual-Band Wi-Fi Based on Gaussian Process Regression and K-Nearest Neighbor. ISPRS Int. J. Geo-Inf. 2021, 10, 706 https://doi.org/10.3390/ijgi10100706

Academic Editors: Guenther Retscher, Ondrej Krejcar, Vassilis Gikas, Michal Kačmařík and Wolfgang Kainz

Received: 24 August 2021

Accepted: 13 October 2021

Published: 15 October 202

Publisher's Note: MDPI stays neutral with regard to jurisdictional claims in published maps and institutional affiliations.

Copyright: (c) 2021 by the authors. Licensee MDPI, Basel, Switzerland. This article is an open access article distributed under the terms and conditions of the Creative Commons Attribution (CC BY) license (https:// creativecommons.org/licenses/by/ $4.0 /)$

\begin{abstract}
Since many Wi-Fi routers can currently transmit two-band signals, we aimed to study dualband Wi-Fi to achieve better positioning results. Thus, this paper proposes a fingerprint positioning method for dual-band Wi-Fi based on Gaussian process regression (GPR) and the K-nearest neighbor $(\mathrm{KNN})$ algorithm. In the offline stage, the received signal strength (RSS) measurements of the $2.4 \mathrm{GHz}$ and $5 \mathrm{GHz}$ signals at the reference points (RPs) are collected and normalized to generate the online dual-band fingerprint, a special fingerprint for dual-band Wi-Fi. Then, a dual-band fingerprint database, which is a dedicated fingerprint database for dual-band $\mathrm{Wi}-\mathrm{Fi}$, is built with the dualband fingerprint and the corresponding RP coordinates. Each dual-band fingerprint constructs its positioning model with the GPR algorithm based on itself and its neighborhood fingerprints, and its corresponding RP coordinates are the label of this model. The neighborhood fingerprints are found by the spatial distances between RPs. In the online stage, the measured RSS values of dual-band $\mathrm{Wi}-\mathrm{Fi}$ are used to generate the online dual-band fingerprint and the $5 \mathrm{GHz}$ fingerprint. Due to the better stability of the $5 \mathrm{GHz}$ signal, an initial position is solved with the $5 \mathrm{GHz}$ fingerprint and the KNN algorithm. Then, the distances between the initial position and model labels are calculated to find a positioning model with the minimum distance, which is the optimal positioning model. Finally, the dual-band fingerprint is input into this model, and the output of this model is the final estimated position. To evaluate the proposed method, we selected two scenarios (A and B) as the test area. In scenario $\mathrm{A}$, the mean error (ME) and root-mean-square error (RMSE) of the proposed method were 1.067 and $1.331 \mathrm{~m}$, respectively. The ME and RMSE in scenario B were 1.432 and $1.712 \mathrm{~m}$, respectively. The experimental results show that the proposed method can achieve a better positioning effect compared with the KNN, Rank, Coverage-area, and GPR algorithms.
\end{abstract}

Keywords: dual-band fingerprint; Wi-Fi; normalization; Gaussian process regression

\section{Introduction}

Indoor positioning has attracted extensive attention due to its broad economic outlook. It can be realized with ultra-wideband (UWB) [1,2], Bluetooth [3,4], Wireless Fidelity (Wi-Fi) [5-10], Radio-Frequency Identification (RFID) [11], Computer Vision [12], Ultrasonic [13], Inertial Navigation System (INS) [14], pseudolite [15], the geomagnetic field $[16,17]$, etc. Among them, Wi-Fi positioning technology is a popular technology due to its lower cost. It mainly includes two categories: received signal strength (RSS) [18] and Channel State Information (CSI) [19]. The CSI-based positioning method requires special equipment, thus hindering its widespread use. In contrast, since most mobile devices, such as smartphones and computers, support the Wi-Fi protocol, the RSS measurement can be easily obtained. In addition, the widely used Wi-Fi routers also create a good environment 
for the acquisition of RSS. Hence, Wi-Fi RSS-based indoor positioning is the most used indoor localization method.

The single-band Wi-Fi signal has been extensively studied in previous works, and the most used band is $2.4 \mathrm{GHz}$. However, the Wi-Fi signals currently have two bands, $2.4 \mathrm{GHz}$ and $5 \mathrm{GHz}$, and most Wi-Fi devices in the market can already transmit two-band signals. These changes in Wi-Fi technology introduce some challenges to Wi-Fi RSS-based positioning technology. It is thus necessary to study how to use dual-band Wi-Fi instead of single-band Wi-Fi to realize higher-accuracy indoor positioning. However, there are few positioning studies based on dual-band Wi-Fi.

Thus, this paper proposes a fingerprint positioning method for dual-band Wi-Fi based on GPR and KNN. This method includes two stages: offline and online. In the offline stage, the RSS measurements of $2.4 \mathrm{GHz}$ and $5 \mathrm{GHz}$ signals are collected to perform the normalization operation. The dual-band fingerprints are generated with the normalized RSS measurements. The dual-band fingerprints and their corresponding RP coordinates comprise a special fingerprint database for dual-band Wi-Fi, i.e., the dual-band fingerprint database. Then, based on the given neighborhood radius, the neighborhood fingerprints of each dual-band fingerprint are searched to construct the positioning model. Finally, all models are stored in the positioning model database, which is used for localization.

In the online stage, the RSS measurements of dual-band signals are collected at the same time to generate the online dual-band fingerprint and $5 \mathrm{GHz}$ fingerprint. Based on the $5 \mathrm{GHz}$ fingerprint and $\mathrm{KNN}$ algorithm, an initial position is solved to obtain an optimal positioning model from the model database. Then, the online dual-band fingerprint is regarded as the input data of the selected positioning model. The output of the positioning model is the final estimated position.

The contributions of this work can be listed as follows:

(1) In this paper, we propose a dedicated dual-band fingerprint for dual-band Wi-Fi, which is generated by the normalized values of the RSS measurements of the 2.4 and $5 \mathrm{GHz}$ signals. The dual band can make full use of existing positioning information and achieve a better positioning effect than the $2.4 \mathrm{GHz}, 5 \mathrm{GHz}$, and hybrid fingerprints. The function of the normalization algorithm is to eliminate the metrics of the RSS measurements to avoid their influence on the value calculation.

(2) A model construction method is used to build the positioning model of each dualband fingerprint in this paper. In the proposed method, based on the GPR algorithm and neighborhood fingerprints, the positioning model of the dual-band fingerprint can be constructed. The proposed model construction method can avoid the decreases in the positioning model's precision as the positioning area increases.

(3) We propose a two-step positioning strategy considering the calculation amount and positioning effect. First, the $5 \mathrm{GHz}$ fingerprint is used to solve a relatively highprecision initial position due to the better stability of the $5 \mathrm{GHz}$ signal than the 2.4 GHz signal, and KNN with low complexity is chosen as the positioning algorithm. Then, the optimal positioning model can be chosen based on the initial position, which is employed to ensure a more accurate position.

\section{Related Work}

The RSS-based positioning method mainly includes ranging [20] and fingerprinting [21]. The ranging method applies the path loss model to estimate the distances between the access points (APs) and the positioning terminal. The localization algorithms, such as the least-square (LS) [22] and trilateration [23] algorithms, solve the positioning result using the estimated distances. However, the ranging has a poorer positioning effect than fingerprinting since the RSS measurement is easily affected. The fingerprinting mainly includes two stages: offline and online. The main task of the offline stage is to construct the fingerprint database. In the online stage, the main purpose is to utilize the positioning algorithms, such as the K-nearest neighbor (KNN) [24-26], neural network (NN) [27], Gaus- 
sian process regression (GPR) [5,28], Horus [29], Rank [30-32], and Coverage-area [33,34] algorithms, to estimate the position of the terminal.

Many methods have been proposed to enhance positioning accuracy since the invention of $\mathrm{Wi}-\mathrm{Fi}$ fingerprinting. The clustering algorithm was introduced to the $\mathrm{Wi}-\mathrm{Fi}$ fingerprint positioning to improve the positioning accuracy and decrease the computation. Machine learning and deep learning were applied to the field of Wi-Fi fingerprint positioning to improve the positioning effect. Interpolation and Crowdsourcing were employed to assist the fast construction of the fingerprint database. However, most of the current research studies on Wi-Fi fingerprint positioning are based on $2.4 \mathrm{GHz} \mathrm{Wi}-\mathrm{Fi}$, while few studies have focused on $5 \mathrm{GHz}$ and dual-band Wi-Fi.

For example, [35] proved that the $5 \mathrm{GHz}$ RSS is more stable than $2.4 \mathrm{GHz}$ RSS. The performance of the fingerprint positioning of the $5 \mathrm{GHz} \mathrm{Wi}-\mathrm{Fi}$ was obviously better than that of 2.4 GHz Wi-Fi [36]. The authors of [37] achieved indoor positioning with the path loss model and the RSS measurements of dual-band Wi-Fi. However, the researchers only studied the range-based positioning and neglected fingerprint-based positioning. More importantly, the ranging precision was not ideal. Literature [38] utilized the RSS measurements of the $5 \mathrm{GHz}$ signals to construct the fingerprint database and obtained a better positioning effect than the $2.4 \mathrm{GHz}$ signals. However, it wasted the existing positioning information, namely the RSS measurements of the $2.4 \mathrm{GHz}$ signals. The authors of [39] distinguished the NLOS and LOS environment using the $2.4 \mathrm{GHz}$ and $5 \mathrm{GHz}$ RSS measurements and employed the capsule networks to derive the position.

\section{Algorithm}

\subsection{Normalization Algorithm}

The normalization algorithm is an approach that transforms a dimensional expression into a non-dimensional expression. It can adjust the data with different metrics to the same metric.

Generally, the normalization algorithm usually maps data between 0 and 1 . Its computation method can be presented as:

$$
R S S_{\text {nor }}=\frac{R S S-R S S_{\min }}{R S S_{\max }-R S S_{\min }}
$$

where $R S S_{\text {nor }}, R S S, R S S_{\text {min }}$, and $R S S_{\text {max }}$ present the normalized RSS value, the measured RSS, the minimum RSS value, and the maximum RSS value, respectively.

\subsection{KNN Algorithm}

The KNN algorithm is a common fingerprint positioning algorithm. It uses the distances between online and offline fingerprints to find K-nearest reference points (RPs) to realize the location estimation. The Euclidean distance, Manhattan distance, cosine distance, and other values can be used for the KNN algorithm. In this paper, we used the Euclidean distance to compute the distances between the online and offline fingerprints. The algorithm steps are as follows:

Step (1): Traverse the fingerprint database and calculate the distances between the online fingerprint and fingerprints in the fingerprint database.

Step (2): Sort these distances and find the K-nearest RPs with minimum distances.

Step (3): Calculate the mean of the K-nearest RPs as the positioning result. The calculation method can be expressed as:

$$
\left(X_{k n n}, Y_{k n n}\right)=\frac{\sum_{i=1}^{K}\left(x_{i}, y_{i}\right)}{K}
$$

Here, $\left(X_{k n n}, Y_{k n n}\right)$ is the estimated location and $\left(x_{i}, y_{i}\right)$ denotes the position of the $i$ th nearest RP. 


\subsection{GPR Algorithm}

In this paper, we utilized the GPR algorithm to construct the positioning model of each dual-band fingerprint. The dual-band fingerprints are training data, which can be expressed as:

$$
R_{i}=\left(R S S_{1}^{i}, R S S_{2}^{i}, R S S_{3}^{i}, \cdots, R S S_{M}^{i}\right)
$$

where $R_{i}$ represents the $i$ th dual-band fingerprint, $R S S_{j}^{i}$ presents the feature of the $j$ th dual-band $\mathrm{Wi}-\mathrm{Fi}$, and $M$ is the number of dual-band Wi-Fi.

In this paper, the ideal output of the model is the position that corresponds to the dual-band fingerprint, which can be expressed as:

$$
L=\left(l_{1,}, l_{2}, l_{3,}, \cdots, l_{N}\right), \quad l_{i}=\left(x_{i}, y_{i}\right)
$$

where $N$ is the number of RPs, which is also the amount of the dual-band fingerprints used to build the positioning model, $L$ denotes the RP coordinate collection in the model construction, and $\left(x_{i}, y_{i}\right)$ represents the coordinates of the $i$ th RP.

Following the GPR algorithm, there is a mapping between the input and output data, as illustrated in Equation (5):

$$
l_{i}=f\left(R_{i}\right)+\gamma
$$

where $\gamma$ follows a Gaussian distribution, namely $\gamma \sim N\left(0, \sigma^{2}\right)$, and $l_{i}$ represents the coordinates of the $i$ th RP.

The main purpose of model training is to find the potential relation between the input and output data. GPR can solve the potential mapping relation of the input and output data. The Gaussian process is a set of random variables that are subject to a joint Gaussian distribution, which is determined by a mean function and covariance function, as shown in Equation (6):

$$
f(R) \sim G P(m(R), K(R, R))
$$

where $m(R)$ represents the mean function that can be set as zero without loss of generality, $K(R, R)$ denotes the covariance matrix, $k\left(R_{i}, R_{j}\right)$ presents the covariance function that can be calculated by Equation (9), and $f(R)$ represents the Gaussian process.

$$
\begin{gathered}
m(R)=E[f(R)] \\
K(R, R)=\left[\begin{array}{cccc}
k\left(R_{1}, R_{1}\right) & k\left(R_{1}, R_{2}\right) & \ldots & k\left(R_{1}, R_{N}\right) \\
k\left(R_{2}, R_{1}\right) & k\left(R_{2}, R_{2}\right) & \cdots & k\left(R_{2}, R_{N}\right) \\
& \vdots & \ddots & \vdots \\
k\left(R_{N}, R_{1}\right) & k\left(R_{N}, R_{2}\right) & \cdots & k\left(R_{N}, R_{N}\right)
\end{array}\right] \\
k\left(R_{i}, R_{j}\right)=E\left[\left(f\left(R_{i}\right)-m\left(R_{i}\right)\right)\left(f\left(R_{j}\right)-m\left(R_{j}\right)\right)\right]
\end{gathered}
$$

Here, $E(\cdot)$ indicates the expectation operator. The main purpose of model training is to solve the hyperparameters $\left[\sigma, \delta_{f}, \lambda_{l}\right] . \delta_{f}$ represents the signal standard deviation, $\lambda_{l}$ is the length-scale parameter, and $R_{i}$ presents the $i$ th dual-band fingerprint.

In this paper, the Euclidean distance was selected to calculate $k\left(R_{i}, R_{j}\right)$, which is represented by $\left\|R_{i}-R_{j}\right\| . k\left(R_{i}, R_{j}\right)$ was calculated by the kernel function, as shown in Equation (10):

$$
k\left(R_{i}, R_{j}\right)=\delta_{f}^{2} \exp \left(-\frac{\left\|R_{i}-R_{j}\right\|^{2}}{2 \lambda_{l}^{2}}\right)
$$

The prediction position $l_{*}$ and training positions $L$ follow a multivariate Gaussian distribution jointly as follows:

$$
\left[\begin{array}{c}
L \\
l_{*}
\end{array}\right]=N\left\{0,\left[\begin{array}{cc}
K(R, R) & K\left(R, R_{*}\right) \\
K\left(R_{*}, R\right) & K\left(R_{*}, R_{*}\right)
\end{array}\right]\right\}
$$


where $R_{*}$ and $R$ are the test data and training data, respectively. The posterior distribution $P\left(l_{*} \mid L\right)$ can be denoted as:

$$
l_{*} \mid L=N \sim\left(K\left(R_{*}, R\right) K(R, R)^{-1} L, K\left(R_{*}, R\right)-K\left(R_{*}, R\right) K(R, R)^{-1} K\left(R, R_{*}\right)\right)
$$

In the positioning estimation stage, the collected RSS measurements of dual-band Wi-Fi are utilized to produce the dual-band fingerprint, which is regarded as the input data of the positioning model. The output of the model is the estimated position.

\subsection{Rank Algorithm}

The Rank algorithm is a fingerprinting algorithm based on the rankings of RSS measurements, which can avoid the influence of device heterogeneity. In this Rank algorithm, the rankings of RSS values of the offline and online stages were used to construct the fingerprint database and solve the positioning results.

Suppose that there is a set of RSS values, $R_{i}=\left(R S S_{1}^{i}, R S S_{2}^{i}, R S S_{3}^{i}, \cdots, R S S_{M}^{i}\right)$. The fingerprint of the Rank algorithm can be denoted as:

$$
R_{\text {Rank }}^{i}=\operatorname{Sort}\left(R_{i}\right)=\left(d_{1}, d_{2}, d_{3}, \cdots, d_{M}\right)
$$

where $R_{\text {Rank }}^{i}$ represents the fingerprint of the Rank algorithm, $d_{i}$ is the rank value of the $i$ th RSS measurements at descending order, and $M$ is the number of RSS measurements.

Then, the similarities between the online and offline rank vectors were calculated to find K nearest RPs with minimum similarities, which were used to solve the location. In the Rank algorithm, the spearman distance, Jaccard coefficient, hamming distance, Canberra distance, and other values can be used to compute the similarity.

\subsection{Coverage-Area Algorithm}

The Coverage-area algorithm uses the MACs of the scanned APs to achieve the positioning. Its offline fingerprint can be expressed as:

$$
R_{\text {coverage }}^{i}=\left(\left(x_{i}, y_{i}\right), M A C_{1}, M A C_{2}, M A C_{3}, \cdots, M A C_{M}\right)
$$

where $R_{\text {coverage }}^{i}$ represents the fingerprint of the Coverage-area algorithm, and $M A C_{i}$ is the $\mathrm{MAC}$ of the $i$ th $\mathrm{AP}$, and $M$ is the number of scanned APs.

In the online stages, there is a set of MACs, $M A C_{\text {scan }}^{i}=\left(M A C_{1}, M A C_{2}, M A C_{3}, \cdots\right.$, $\left.M A C_{M}\right)$, which is the online fingerprint of the Coverage-area algorithm. Based on the MAC scanned online, the shared MAC of the offline and online fingerprints can be obtained. The RPs with the most shared MACs were chosen to estimate the position.

\section{The Proposed Method}

\subsection{Stability Analysis of RSS Measurements of Dual-Band Wi-Fi}

Since dual-band Wi-Fi has different bands, there should be differences between the two RSS measurements. In this section, we analyze the stability of RSS measurements of two-band signals. The RSS measurements of the $2.4 \mathrm{GHz}$ and $5 \mathrm{GHz}$ signals were gathered to study their stability, as shown in Figure 1. To conduct this experiment, the experimenter stood in a fixed position and gathered the RSS measurements of a dual-band Wi-Fi router from four directions. The collection frequency was $1 \mathrm{~Hz}$, and the original RSS measurements of each direction were employed to analyze the stability of the 2.4 and $5 \mathrm{GHz}$ signals. 


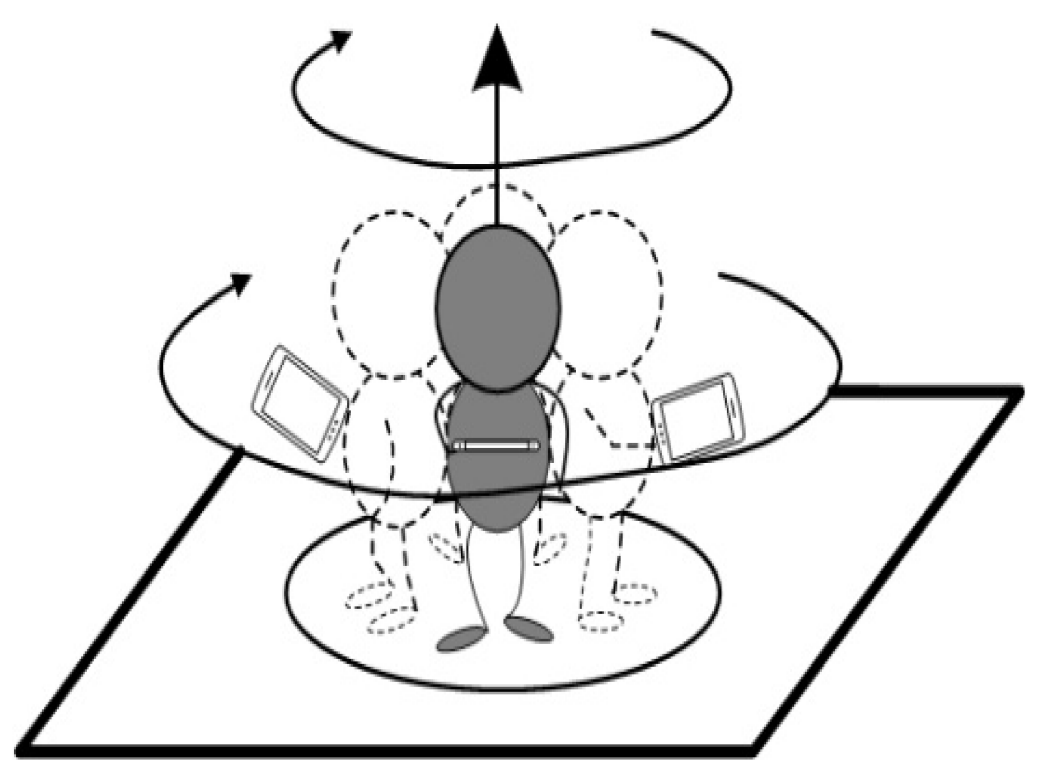

Figure 1. The schematic diagram of the RSS measurement data collection.

The original RSS measurements of each scan of the four orientations are shown in Figure 2. In each direction, the stability of the RSS measurements of the $2.4 \mathrm{GHz}$ and $5 \mathrm{GHz}$ signals was different. The RSS measurements of the $5 \mathrm{GHz}$ signals were more stable than those of the $2.4 \mathrm{GHz}$ signals. For example, the RSS measurements of the $2.4 \mathrm{GHz}$ signals had a few strong jumps at orientations 1 and 2 . Therefore, it is necessary to study how to appropriately utilize the RSS measurements of dual-band Wi-Fi to achieve a better positioning effect than single-band Wi-Fi.

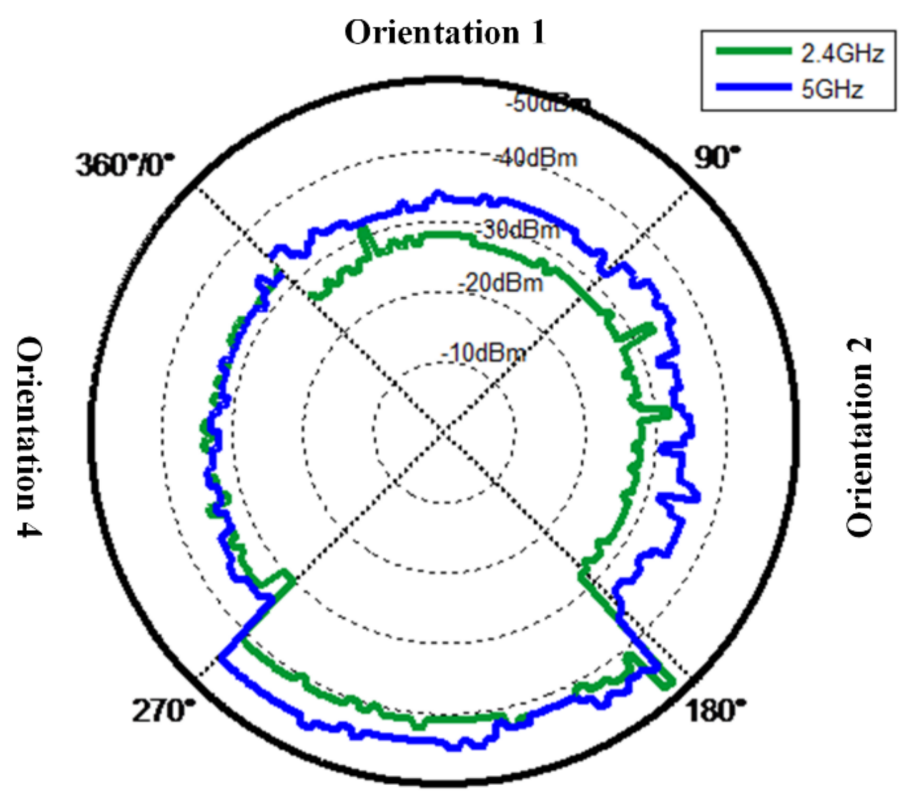

Orientation 3

Figure 2. The RSS measurements of the $2.4 \mathrm{GHz}$ and $5 \mathrm{GHz}$ signals.

\subsection{The Traditional Fingerprint}

Dual-band Wi-Fi can transmit $2.4 \mathrm{GHz}$ and $5 \mathrm{GHz}$ signals, meaning that more available positioning information (2.4 GHz and $5 \mathrm{GHz}$ RSS) can be obtained when dual-band Wi-Fi is utilized for indoor positioning compared with single-band Wi-Fi. Generally, the RSS measurements of any band Wi-Fi can be employed to construct the fingerprint database. In 
other words, we can utilize the RSS values of the $2.4 \mathrm{GHz}$ and $5 \mathrm{GHz}$ signals to generate the $2.4 \mathrm{GHz}$ and $5 \mathrm{GHz}$ fingerprints, respectively, as shown in Figure 3, where $M A C$ represents the media access control (MAC) address of AP, and superscript and subscript notations are the serial number and signal band, respectively, and $M$ denotes the number of the perceived APs.

\begin{tabular}{lllllll}
\hline$M A C_{2.4 G H z}^{I}$ & $M A C_{2.4 G H z}^{2}$ & $M A C_{2.4 G H z}^{3}$ & $M A C_{2.4 G H z}^{4}$ & $\cdots$ & $M A C_{2.4 G H z}^{M-1}$ & $M A C_{2.4 G H z}^{M}$ \\
\hline$R S S_{2.4 G H z}^{1}$ & $R S S_{2.4 G H z}^{2}$ & $R S S_{2.4 G H z}^{3}$ & $R S S_{2.4 G H z}^{4}$ & $\cdots$ & $R_{S S}^{M-1}$ & $R S S_{2.4 G H z}^{M}$ \\
\hline
\end{tabular}

(2.4 GHz fingerprint)

\begin{tabular}{|c|c|c|c|c|c|c|}
\hline $\begin{array}{c}M A C_{5 G H z}^{I} \\
\operatorname{RSS}_{5 G H z}^{I}\end{array}$ & $\begin{array}{c}M A C_{5 G H z}^{2} \\
R S S_{5 G H z}^{2}\end{array}$ & $\begin{array}{c}M A C_{5 G H z}^{3} \\
\operatorname{RSS}_{5 G H z}^{3}\end{array}$ & $\begin{array}{c}M A C_{5 G H z}^{4} \\
\operatorname{RSS}_{5 G H z}^{4}\end{array}$ & $\ldots$ & $\begin{array}{c}M A C_{5 G H z}^{M-1} \\
\operatorname{RSS}_{5 G H z}^{M-1}\end{array}$ & $\begin{array}{c}M A C_{5 G H z}^{M} \\
R_{S S_{5 G H z}^{M}}^{M}\end{array}$ \\
\hline
\end{tabular}

( 5 GHz fingerprint)

\begin{tabular}{lllllll}
\hline$M A C_{2.4 G H z}^{I}$ & $M A C_{5 G H z}^{I}$ & $M A C_{2.4 G H z}^{2}$ & $M A C_{5 G H z}^{2}$ & $\cdots$ & $M A C_{2.4 G H z}^{M}$ & $M A C_{5 G H z}^{M}$ \\
\hline$R S S_{2.4 G H z}^{1}$ & $R S S_{5 G H z}^{I}$ & $R S S_{2.4 G H z}^{2}$ & $R S S_{5 G H z}^{2}$ & $\cdots$ & $R S S_{2.4 G H z}^{M}$ & $R S S_{5 G H z}^{M}$ \\
\hline
\end{tabular}

(Hybrid fingerprint)

Figure 3. The traditional fingerprints.

\subsection{The Proposed Dual-Band Fingerprint}

Given that the RSS measurements of the $2.4 \mathrm{GHz}$ and $5 \mathrm{GHz}$ signals have different metrics, it is necessary to adjust two RSS measurements to the same metric. In this paper, the normalization algorithm was utilized to preprocess the two RSS measurements, as shown in Equation (15).

$$
R S S_{n o r}^{i}=\operatorname{Nor}\left(R S S_{2.4 G H z}^{i}\right)+\operatorname{Nor}\left(R S S_{5 G H z}^{i}\right)
$$

where $\operatorname{Nor}\left(R S S_{2.4 \mathrm{GHz}}^{i}\right)$ and $\operatorname{Nor}\left(R S S_{5 \mathrm{GHz}}^{i}\right)$ are the normalized values of the $2.4 \mathrm{GHz}$ and $5 \mathrm{GHz}$ RSS of the ith dual-band Wi-Fi, respectively, and $R S S_{\text {nor }}^{i}$ denotes the feature of the ith dual-band Wi-Fi.

In other words, the RSS measurements of the $2.4 \mathrm{GHz}$ and $5 \mathrm{GHz}$ signals of one dual-band Wi-Fi can generate a feature that represents this dual-band Wi-Fi. Multiple features are used to make up the dual-band fingerprint, as shown in Figure 4.

\begin{tabular}{ccccccc}
\hline$M A C_{2.4 G H z}^{1}$ & $M A C_{2.4 G H z}^{2}$ & $M A C_{2.4 G H z}^{3}$ & $M A C_{2.4 G H z}^{4}$ & $\ldots$ & $M A C_{2.4 G H z}^{M-1}$ & $M A C_{2.4 G H z}^{M}$ \\
$M A C_{5 G H z}^{1}$ & $M A C_{5 G H z}^{2}$ & $M A C_{5 G H z}^{3}$ & $M A C_{5 G H z}^{4}$ & & $M A C_{5 G H z}^{M-1}$ & $M A C_{5 G H z}^{M}$ \\
\hline$R S S_{n o r}^{1}$ & $R S S_{n o r}^{2}$ & $R S S_{\text {nor }}^{3}$ & $R S S_{n o r}^{4}$ & $\ldots$ & $R S S_{n o r}^{M-1}$ & $R S S_{n o r}^{M}$ \\
\hline
\end{tabular}

Figure 4. The dual-band fingerprint.

\subsection{Overview of the Proposed Method}

The proposed method includes two stages, offline and online, as shown in Figure 5. In the offline stage, there were two steps: the fingerprint database construction and positioning model establishment. First, the RSS measurements of the $2.4 \mathrm{GHz}$ and $5 \mathrm{GHz}$ signals at RPs were collected. Then, these measurements were normalized and adjusted to the same metric. Based on these normalized RSS values, the dual-band fingerprints were generated. The dual-band fingerprint database was built with the dual-band fingerprints and their corresponding RP coordinates. Meanwhile, a $5 \mathrm{GHz}$ fingerprint database can be also built with $5 \mathrm{GHz}$ RSS measurements. 

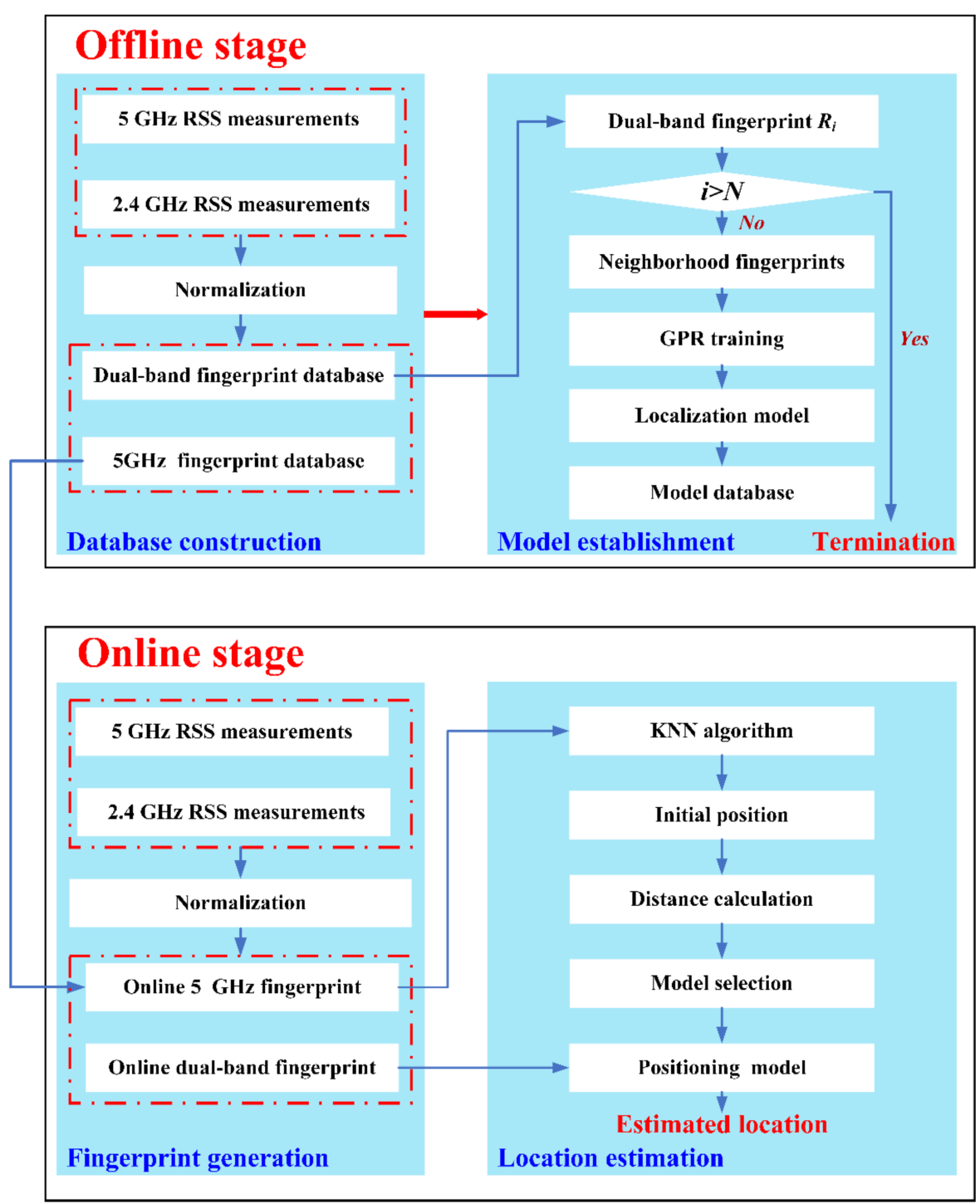

Figure 5. The principle of the proposed method.

Second, we constructed the positioning model. Considering that the positioning model trained by the GPR algorithm has poor accuracy in a larger indoor environment, this paper proposes a novel model construction method that establishes the positioning model of each dual-band fingerprint. In the proposed model construction method, the RP corresponding to the $i$ th dual-band fingerprint $R_{i}$ is seen as a center to search the fingerprint in its neighborhood, namely the neighborhood fingerprint, as shown in Figure 6.

Then, the dual-band fingerprint $R_{i}$ and its neighborhood fingerprints were regarded as the training data to establish a positioning model, and its corresponding RP coordinates were the label of this positioning model. After the positioning model of $R_{i}$ was built, we established the positioning model of the next fingerprint $R_{i+1}$ until all dual-band fingerprints had their positioning models, i.e., $i>N$, as shown in Figure 7. The positioning models of all dual-band fingerprints were stored in a database. 


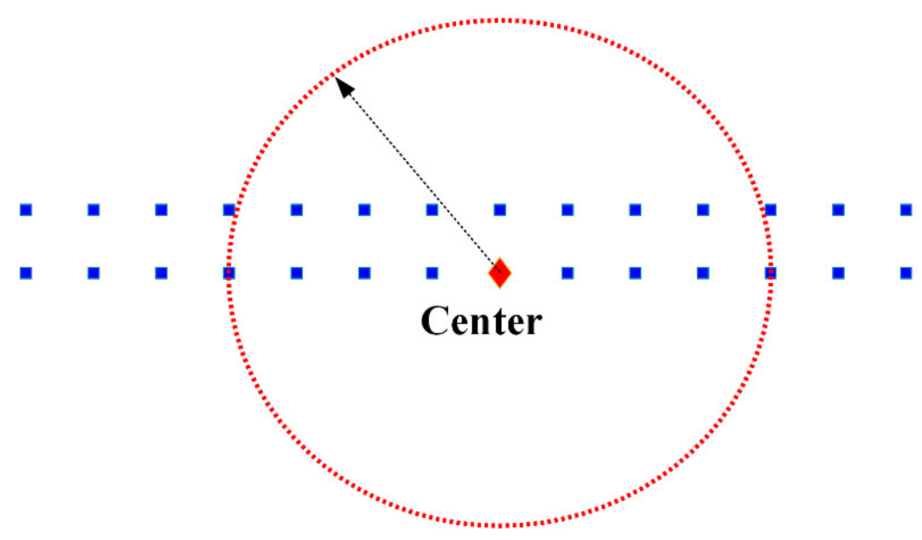

Figure 6. The schematic diagram of the find neighboring fingerprints.

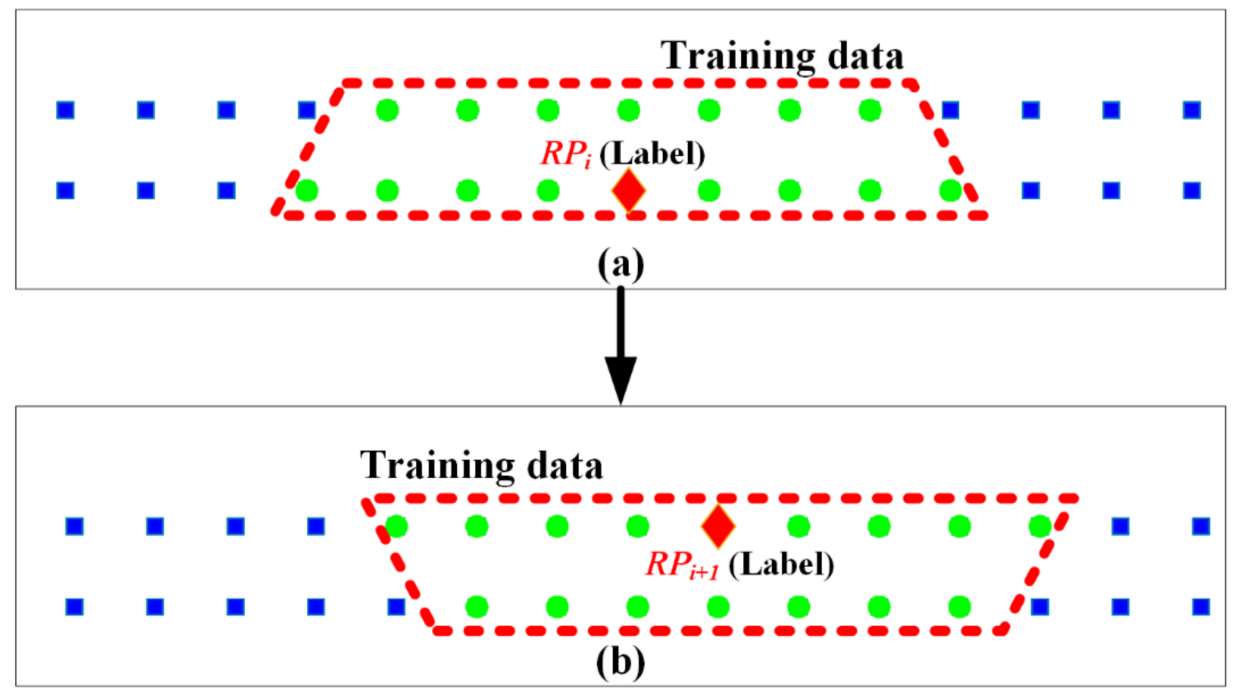

Figure 7. The schematic diagram of the model construction, (a) show the model construction of dual-band fingerprint $R_{i}$, (b) show the model construction of dual-band fingerprint $R_{i+1}$.

In the online stage, the first step is to collect the RSS measurements of the $2.4 \mathrm{GHz}$ and $5 \mathrm{GHz}$ signals to generate the online dual-band and $5 \mathrm{GHz}$ fingerprints. However, to generate the dual-band fingerprint, the RSS measurements must be normalized. The sum of the two sets of normalized RSS values is the dual-band fingerprint.

The second step is the location estimation This step primarily aims to find an optimal positioning model to calculate the position of the terminal. The KNN algorithm is utilized to obtain an initial position based on the $5 \mathrm{GHz}$ fingerprint. Then, the spatial distances between this initial position and model labels are computed, i.e., the distances between this initial position and RPs, which were employed to find an optimal positioning model. The model corresponding to the minimum distance is the final optimal localization model. The dual-band fingerprint is input into the model as input data, and the output is the final location estimation result.

\section{Experimental Analysis}

\subsection{Experimental Environment}

To evaluate the performance of the proposed method, two scenarios, Scenario A and Scenario B, were chosen as the experimental areas, as shown in Figures 8 and 9, respectively. 


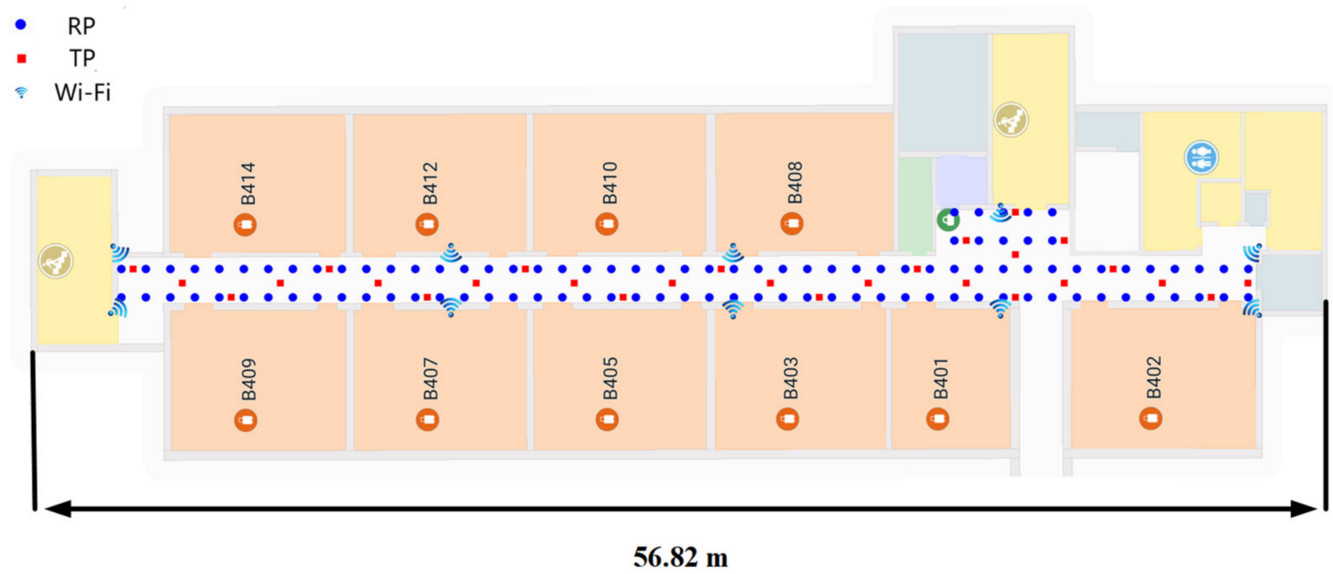

Figure 8. Scenario A.

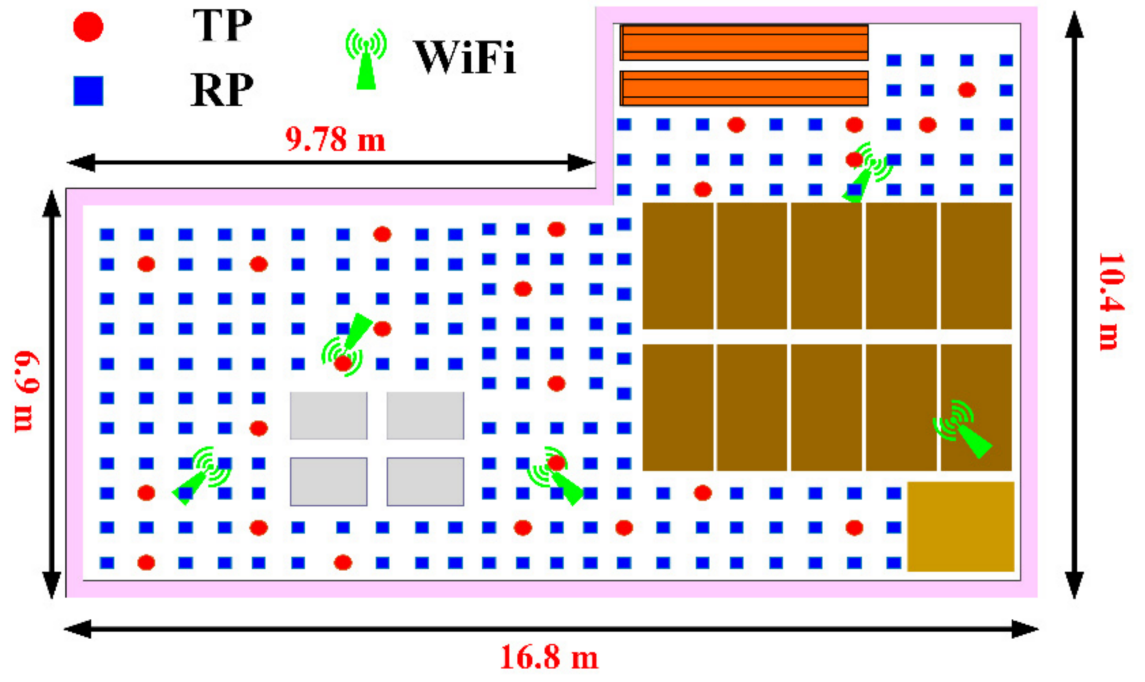

Figure 9. Scenario B.

Scenario A, as shown in Figure 8, had a length of $56.82 \mathrm{~m}$ and an area of approximately $154.37 \mathrm{~m}^{2}$. The blue circle and red rectangle in the Figure represent the RP and test point (TP), respectively. The distance between two adjacent RPs was $1.2 \mathrm{~m}$. There were 10 dual-band Wi-Fi routers in Scenario A, which could all transmit dual-band signals. There were 114 RPs and 28 TPs in Scenario A. The XIAOMI 8 Smartphone was used to gather the data in this scenario.

Scenario B was approximately $140.49 \mathrm{~m}^{2}$, and its length was $16.8 \mathrm{~m}$. The blue rectangle and red circle in Figure 9 represent the RP and TP, respectively. The distance between adjacent RPs was, at maximum, $0.7 \mathrm{~m}$, and the distance between a small number of adjacent RPs was $0.6 \mathrm{~m}$. There were five dual-band Wi-Fi routers with dual-band signal emissions in Scenario B. There were 183 and 24 RPs and TPs in Scenario B, respectively. In this scenario, the HUAWEI P20 Smartphone was used to collect data.

The collection methods of the two scenarios shared the same acquisition frequency and time. The experimenters were based on the RP and collected the RSS measurements of the $2.4 \mathrm{GHz}$ and $5 \mathrm{GHz}$ signals. The acquisition time was $30 \mathrm{~s}$, and the acquisition frequency was $1 \mathrm{~Hz}$. The RSS measurements of the dual-band signals were used to generate the $2.4 \mathrm{GHz}$ fingerprint, $5 \mathrm{GHz}$ fingerprint, hybrid fingerprint, and dual-band fingerprint.

To test the performance of the proposed positioning method, the RSS measurements at each TP were gathered, and the same acquisition method was used as that of RP. However, the acquisition time was $10 \mathrm{~s}$. 


\subsection{Experimental Description}

To assess the performance of the proposed method, three experiments were conducted: the comparison of model precision of the proposed method and GPR; the study of the positioning effect of the $2.4 \mathrm{GHz}, 5 \mathrm{GHz}$, hybrid, and dual-band fingerprints in Scenarios A and $\mathrm{B}$; and the study of the contradistinction between the proposed method and the other classic methods (KNN, Rank, Coverage-area, and GPR).

In this paper, the accuracy is measured with mean error (ME). The root-mean-square error (RMSE) is employed as the index of stability. In addition, the model loss is utilized as the index of model accuracy.

\subsection{Comparison of Model Precision}

To rate the model construction accuracy of the proposed method, Scenario B was used as the experimental area. The model losses of the proposed method and GPR were calculated to evaluate the model construction precision. Since there were multiple models due to the application of the proposed model's construction method, the mean of multiple model losses was the model loss of the proposed method in this paper.

The model losses of the proposed method and GPR are presented in Figure 10. The model losses of the proposed method and GPR were 0.875 and $1.644 \mathrm{~m}$, respectively. Compared with the GPR method, the model accuracy of the proposed method was improved by $46.78 \%$. Thus, the constructed model of the proposed method is better than that of GPR.

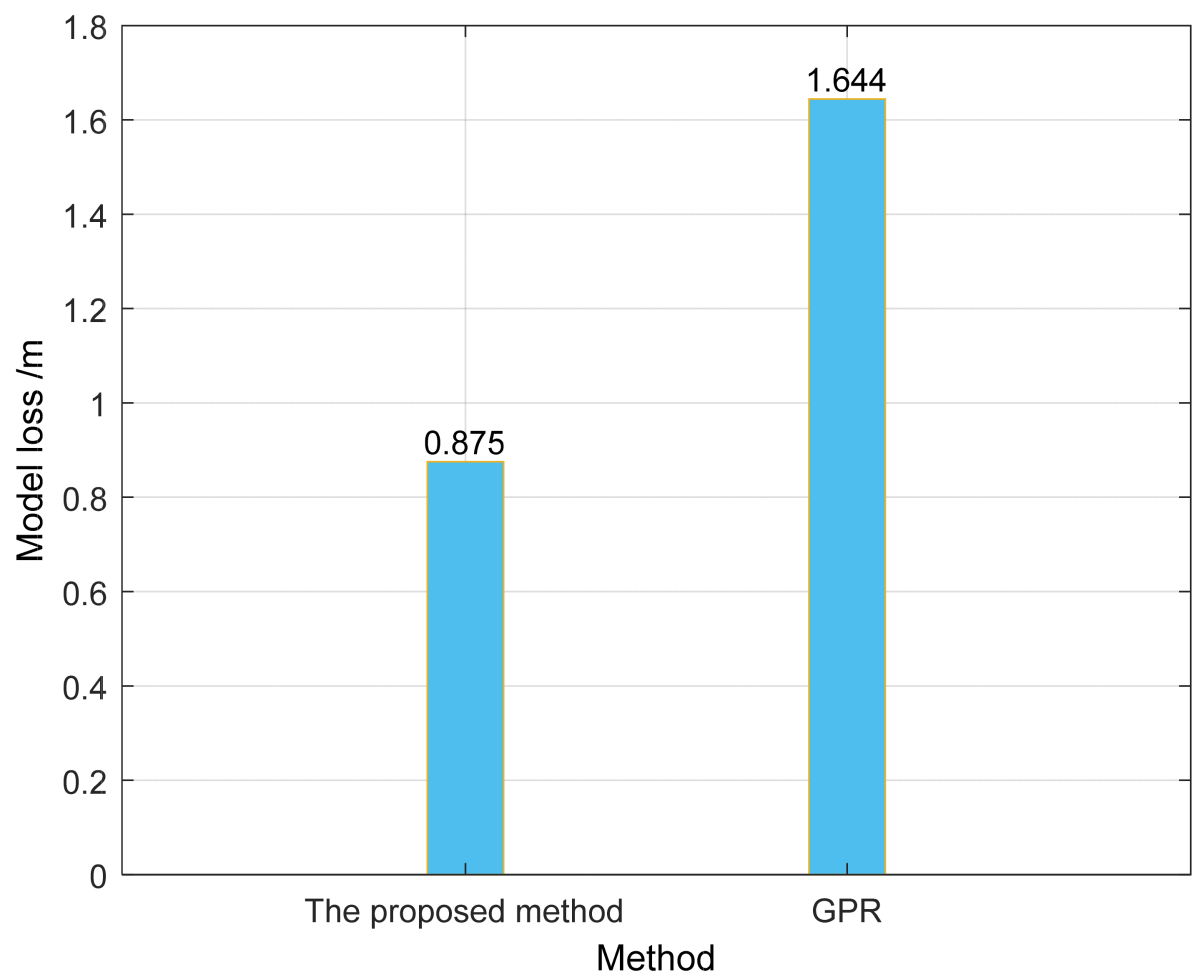

Figure 10. The model losses of the proposed method and GPR.

5.4. Positioning Effect of the $2.4 \mathrm{GHz}, 5 \mathrm{GHz}$, Hybrid, and Dual-Band Fingerprints in Scenario A

In this section, Scenario A was used as a test area to study the positioning effect of the $2.4 \mathrm{GHz}, 5 \mathrm{GHz}$, hybrid, and dual-band fingerprints. The GPR algorithm was chosen as the positioning algorithm to estimate the position. Figure 11 shows the positioning effect of the different fingerprints, mainly presenting the MEs and RMSEs of the $2.4 \mathrm{GHz}, 5 \mathrm{GHz}$, hybrid, and dual-band fingerprints. 


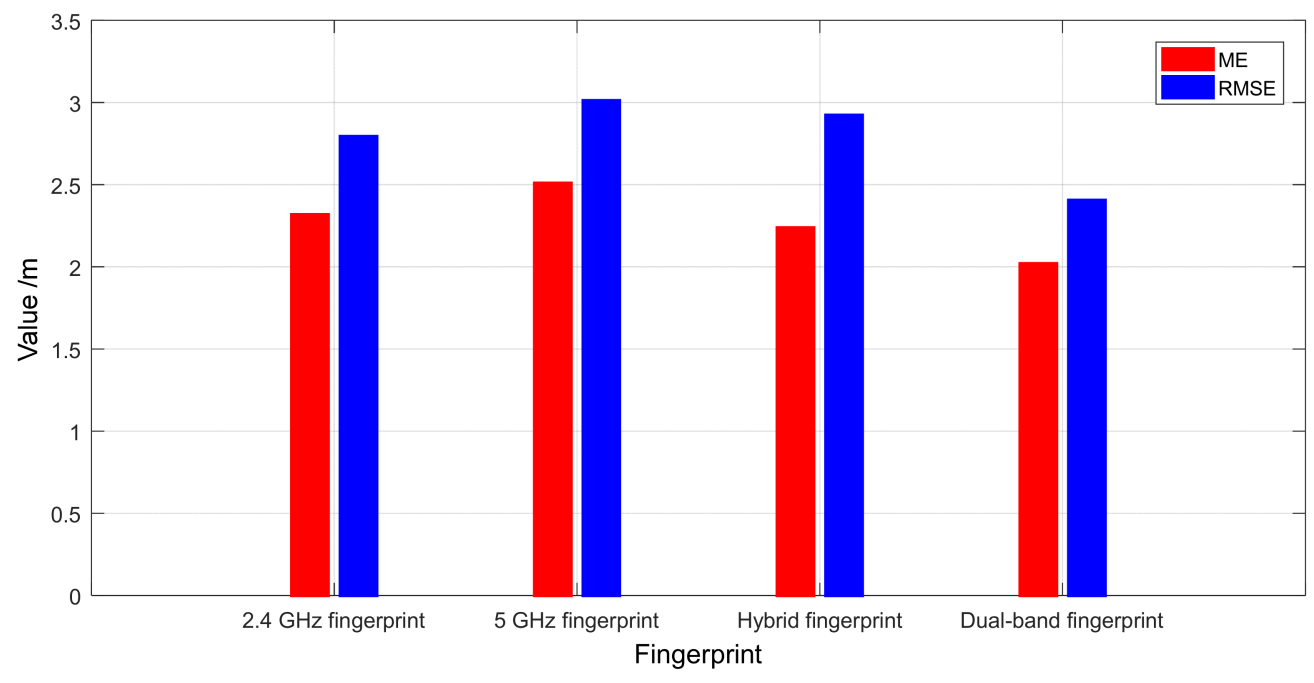

Figure 11. The MEs and RMSEs of the $2.4 \mathrm{GHz}, 5 \mathrm{GHz}$, hybrid, and dual-band fingerprints.

When the GPR algorithm was the positioning algorithm, the MEs of the $2.4 \mathrm{GHz}$, $5 \mathrm{GHz}$, hybrid, and dual-band fingerprints were 2.318, 2.509, 2.238, and $2.020 \mathrm{~m}$, respectively, and RMSEs were 2.793, 3.011, 2.922, and $2.404 \mathrm{~m}$, respectively, as shown in Table 1. We can conclude that the positioning stability and accuracy of hybrid and dual-band fingerprints are better than those of $2.4 \mathrm{GHz}$ and $5 \mathrm{GHz}$ fingerprints.

Table 1. The MEs and RMSEs of the $2.4 \mathrm{GHz}, 5 \mathrm{GHz}$, hybrid, and dual-band fingerprints in Scenario A.

\begin{tabular}{ccc}
\hline Fingerprint & ME & RMSE \\
\hline 2.4 GHz fingerprint & 2.318 & 2.793 \\
5 GHz fingerprint & 2.509 & 3.011 \\
Hybrid fingerprint & 2.238 & 2.922 \\
Dual-band fingerprint & 2.020 & 2.404 \\
\hline
\end{tabular}

The dual-band fingerprint had improvements of 0.66 and $0.218 \mathrm{~m}$ on the RMSE and on ME, respectively, compared to the hybrid fingerprint. The dual-band fingerprint was better than the hybrid fingerprint. The experimental results also indicate that the GPR algorithm would not have a good positioning effect in a large indoor area.

\subsection{Positioning Effect of the $2.4 \mathrm{GHz}, 5 \mathrm{GHz}$, Hybrid, and Dual-Band Fingerprints in Scenario $B$}

In this section, we introduce the positioning effect of the $2.4 \mathrm{GHz}, 5 \mathrm{GHz}$, hybrid, and dual-band fingerprints in Scenario B. KNN was the positioning algorithm. Figure 12 represents the MEs of the $2.4 \mathrm{GHz}, 5 \mathrm{GHz}$, hybrid, and dual-band fingerprints. The positioning effect of the dual-band fingerprint was better than that of the $4 \mathrm{GHz}, 5 \mathrm{GHz}$, and hybrid fingerprints.

Table 2 presents the statistical results of the positioning errors of the $2.4 \mathrm{GHz}, 5 \mathrm{GHz}$, hybrid, and dual-band fingerprints in Scenario B. The ME and RMSE of the $2.4 \mathrm{GHz}$ fingerprint were 2.366 and $2.589 \mathrm{~m}$, respectively, and those of $5 \mathrm{GHz}$ fingerprint were 2.352 and $2.559 \mathrm{~m}$, respectively. The MEs of the hybrid and dual-band fingerprints were $2.12 \mathrm{~m}$ and $1.896 \mathrm{~m}$, respectively, and RMSEs were 2.392 and $2.152 \mathrm{~m}$, respectively.

The ME of the dual-band fingerprint decreased by $0.47,0.456$, and $0.224 \mathrm{~m}$, respectively. The RMSE of the dual-band fingerprint reduced by $0.435,0.405$ and $0.238 \mathrm{~m}$, respectively. Thus, the positioning effect of the dual-band fingerprint is better than that of $2.4 \mathrm{GHz}$, $5 \mathrm{GHz}$, and hybrid fingerprints. 


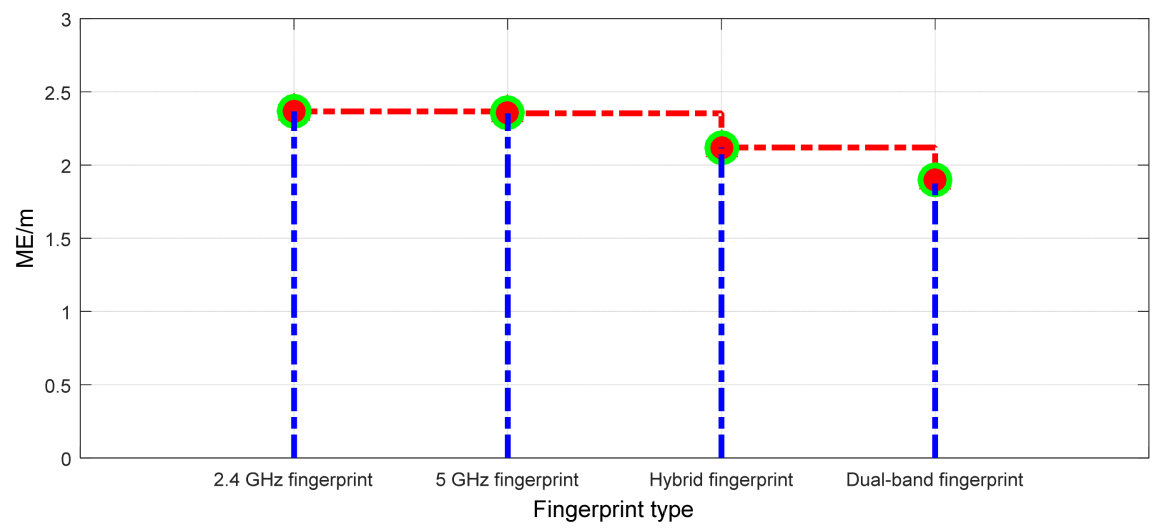

Figure 12. The RMSEs of the $2.4 \mathrm{GHz}, 5 \mathrm{GHz}$, hybrid, and dual-band fingerprints.

Table 2. The MEs and RMSEs of the $2.4 \mathrm{GHz}, 5 \mathrm{GHz}$, hybrid, and dual-band fingerprints in Scenario $\mathrm{B}$.

\begin{tabular}{ccc}
\hline Fingerprint & ME & RMSE \\
\hline 2.4 GHz fingerprint & 2.366 & 2.589 \\
5 GHz fingerprint & 2.352 & 2.559 \\
Hybrid fingerprint & 2.120 & 2.392 \\
Dual-band fingerprint & 1.896 & 2.154 \\
\hline
\end{tabular}

\subsection{Positioning Effect of the Proposed Method in Two Scenarios}

In this section, the performance of the proposed method is analyzed by comparison with the other positioning algorithms (KNN, Rank, Coverage-area, and GPR). Scenario A and $\mathrm{B}$ are regarded as test areas to rate the accuracy and stability of the proposed method, respectively. At first, the proposed method and comparison algorithm were implemented in Scenario A. Then, the positioning errors of the above methods were analyzed, aiming to evaluate the localization performance of the positioning algorithm.

Figure 13 shows the cumulative distribution functions (CDFs) of the positioning errors of KNN, GPR, Rank, Coverage-area, and the proposed method when the experimental area was Scenario A and the dual-band fingerprint was used. The proposed method had the best positioning effect among all methods, with a maximum error of $2.725 \mathrm{~m}$. The maximum errors of KNN, Rank, Coverage-area, and GPR were 6.992, 11.724, 8.824, and $5.183 \mathrm{~m}$, respectively, which indicates that the proposed method has a good ability to avoid positioning errors.

Table 3 presents the statistical results of the positioning errors of KNN, GPR, and the proposed method. The results include the ME and RMSE of each positioning method, as well as the positioning errors corresponding to some important cumulative error probabilities, such as $50 \%, 70 \%$, and $90 \%$.

Table 3. The statistical results of the positioning errors in Scenario A/m.

\begin{tabular}{cccccc}
\hline Method & $\mathbf{5 0 \%}$ & $\mathbf{7 0} \%$ & $\mathbf{9 0} \%$ & ME & RMSE \\
\hline KNN & 1.393 & 2.220 & 3.042 & 1.803 & 2.262 \\
Rank & 2.425 & 4.573 & 7.328 & 3.651 & 4.876 \\
Coverage-area & 2.179 & 3.900 & 6.294 & 3.159 & 3.837 \\
GPR & 1.631 & 2.311 & 3.562 & 2.020 & 2.404 \\
Proposed method & 0.748 & 1.423 & 2.008 & 1.067 & 1.331 \\
\hline
\end{tabular}




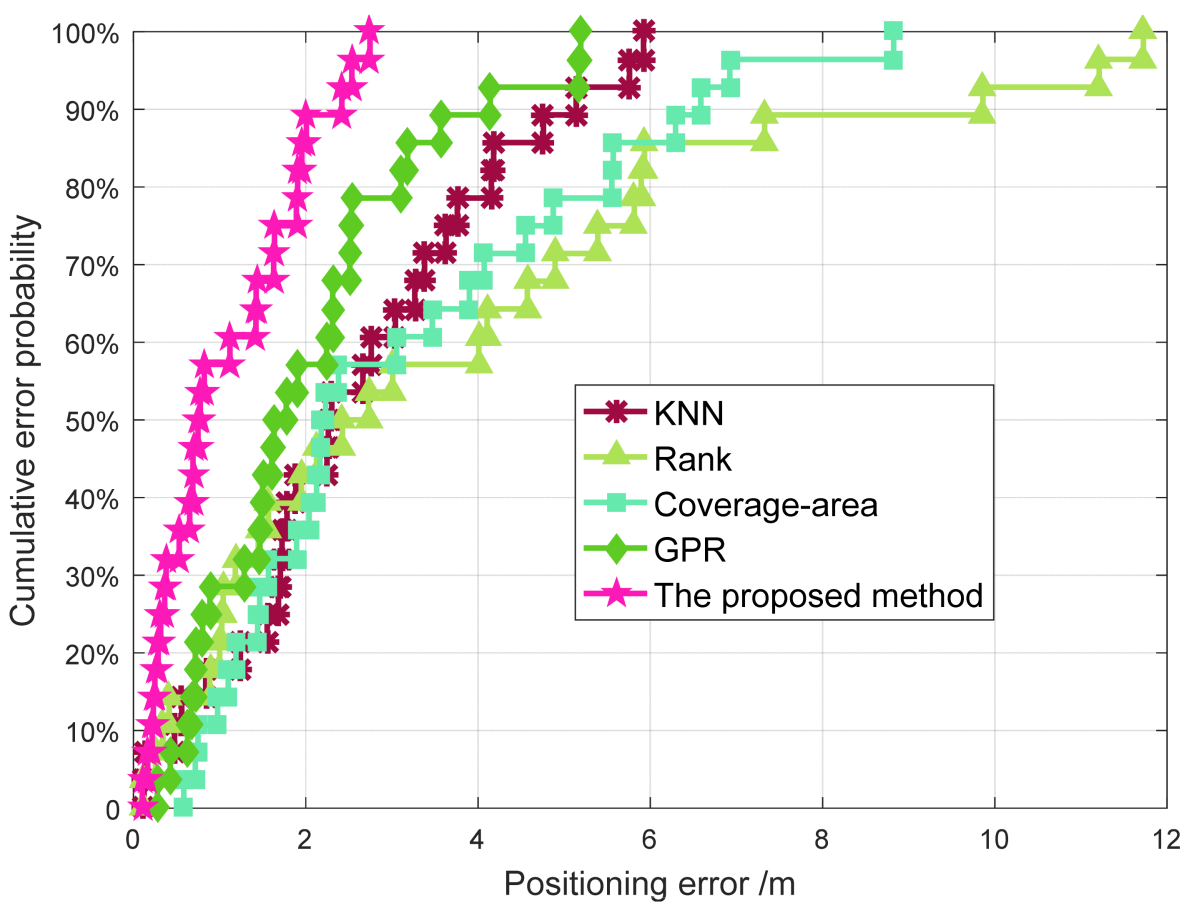

Figure 13. The probability distribution functions of the positioning errors in Scenario A.

The ME and RMSE of the proposed method were $1.067 \mathrm{~m}$ and $1.331 \mathrm{~m}$, respectively. They decreased by $0.736 \mathrm{~m}$ and $0.931 \mathrm{~m}$, respectively, compared with KNN. In addition, compared with GPR, the ME and RMSE decreased by 0.953 and $1.073 \mathrm{~m}$, respectively. Compared with Rank, the accuracy of the proposed method was improved by $70.78 \%$, and the stability was improved by $72.7 \%$. The ME and RMSE of the proposed method were reduced by $66.22 \%$ and $65.31 \%$, respectively, compared to the Coverage-area. Therefore, we can conclude that the proposed method is superior to KNN, Rank, Coverage-area, and GPR.

Based on the experimental results of Scenario A, we can see that the ME of the proposed method decreased by 1.353 and $0.748 \mathrm{~m}$ compared with the $2.4 \mathrm{GHz}$ and $5 \mathrm{GHz}$ fingerprints using KNN. The ME of the proposed method was reduced by 1.251 and $1.442 \mathrm{~m}$ compared to that of the $2.4 \mathrm{GHz}$ and $5 \mathrm{GHz}$ fingerprints using GPR. The positioning effect of the proposed method was better than that of single-band Wi-Fi.

Considering that Scenario A is a corridor and has a simple structure, we chose another scenario, Scenario B, to assess the proposed method. The positioning results of Scenario B are shown in Figure 14, which presents the CDFs of the positioning errors of KNN, Rank, Coverage-area, GPR, and the proposed method. Their maximum errors are 4.692, 7.63, $6.874,4.414$, and $4.244 \mathrm{~m}$, respectively.

Table 4 shows the positioning effect in Scenario B. The proposed method had an ME of $1.432 \mathrm{~m}$, which was decreased by $24.47 \%, 49 \%, 60.37 \%$, and $27.71 \%$ compared to KNN, rank, coverage-area, and GRP, respectively. Meanwhile, compared with KNN, Rank, Coverage-area, and GRP, the RMSE of the proposed method had $20.48 \%, 48.7 \%, 57.7 \%$, and $25.27 \%$ reductions, respectively. The positioning results in Scenario B also prove that the proposed method is better than KNN, Rank, Coverage-area, and GPR.

In this section, we evaluate the positioning performance of the proposed method according to the positioning error in two scenarios. However, the above analysis mainly focuses on a single scenario. Thus, we combined the positioning results of two scenarios to evaluate the positioning performance of the proposed method more comprehensively. 


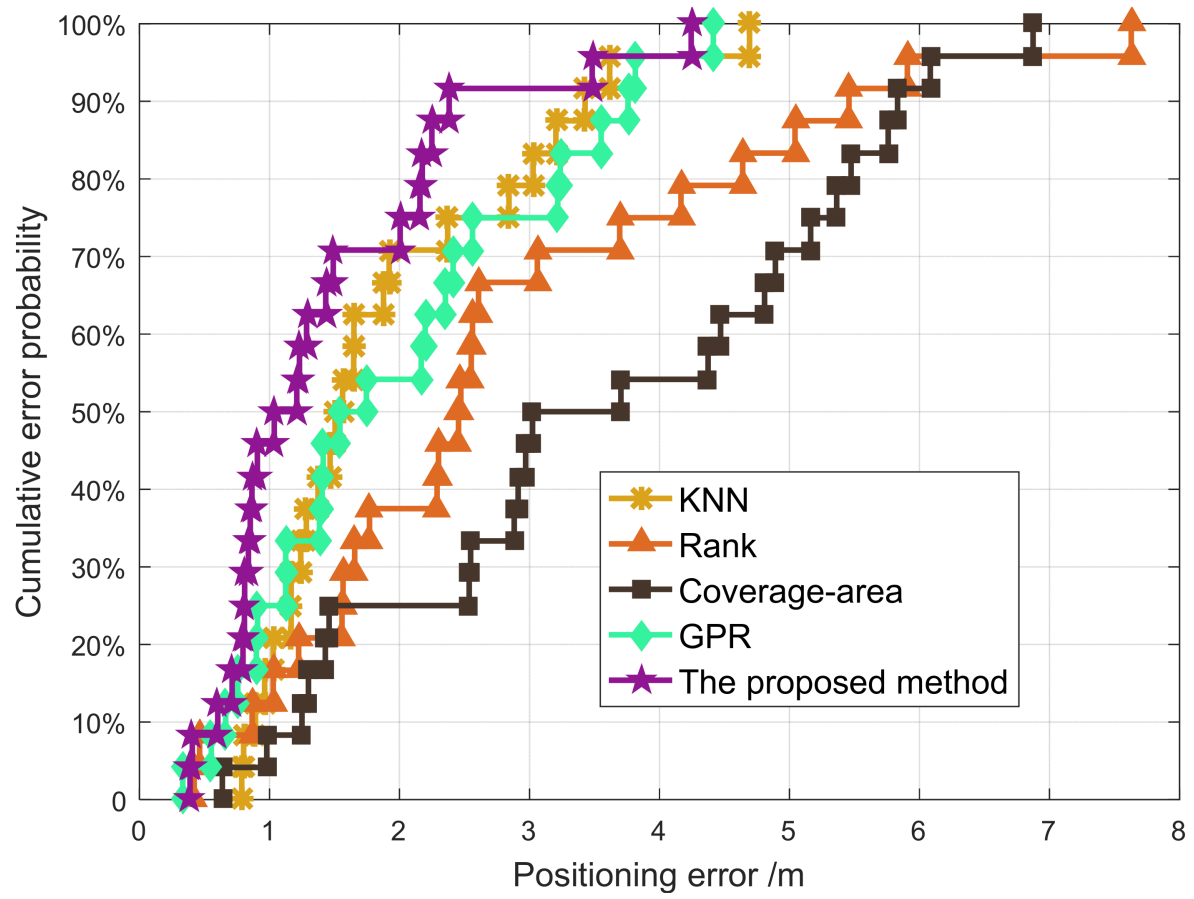

Figure 14. The CDFs of the positioning errors in Scenario A.

Table 4. The statistical results of the positioning errors in Scenario B/m.

\begin{tabular}{cccccc}
\hline Method & $\mathbf{5 0 \%}$ & $\mathbf{7 0} \%$ & $\mathbf{9 0} \%$ & ME & RMSE \\
\hline KNN & 1.504 & 1.876 & 3.204 & 1.896 & 2.153 \\
Rank & 2.453 & 2.608 & 5.042 & 2.808 & 3.337 \\
Coverage-area & 3.023 & 4.808 & 5.760 & 3.613 & 4.047 \\
GPR & 1.542 & 2.354 & 3.551 & 1.981 & 2.291 \\
Proposed method & 1.037 & 1.432 & 2.249 & 1.432 & 1.712 \\
\hline
\end{tabular}

Figure 15 shows the positioning effect of KNN, Rank, Coverage-area, GPR, and the proposed method in two scenarios. The lower quartile, median, upper quartile of positioning errors of the proposed method were better than those of the KNN, Rank, Coverage-area, and GPR algorithms. Both the positioning stability and accuracy of the proposed method were better than those of KNN, Rank, Coverage-area, and GPR.

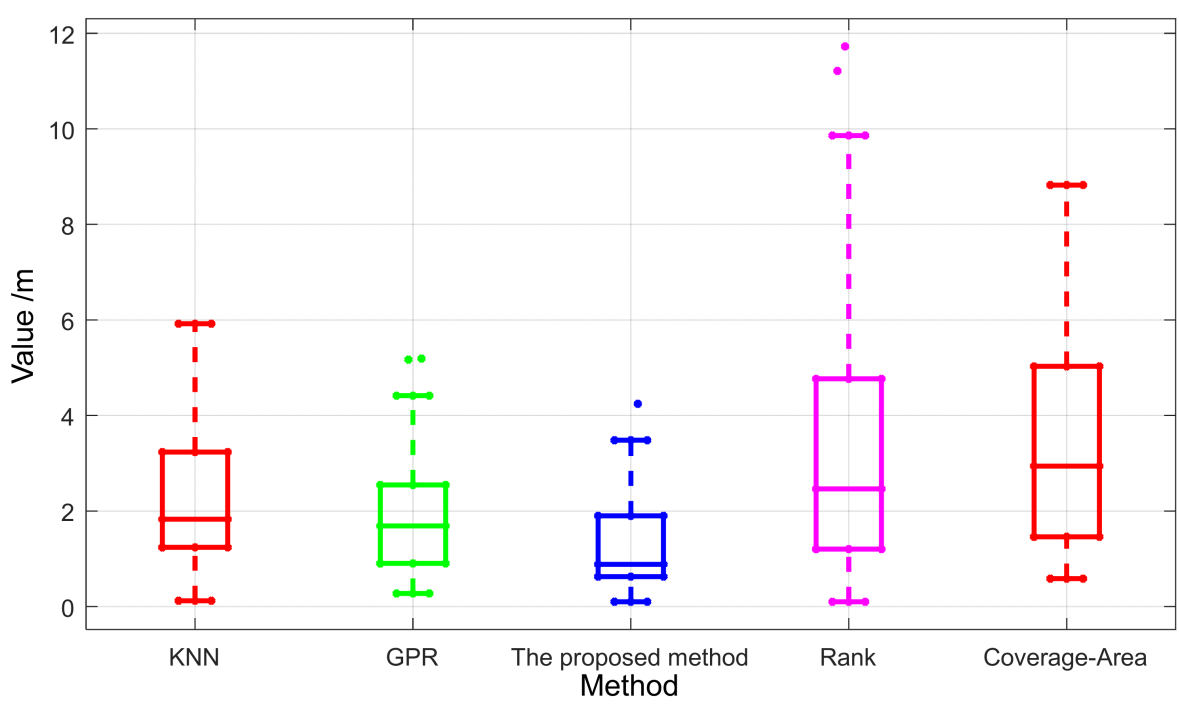

Figure 15. The positioning effect of the proposed method in the two scenarios. 
Based on the results presented in Table 5, we can see that the positioning effect of the proposed method was better than that of KNN, Rank, Coverage-area, and GPR. The positioning stability of the proposed method was improved by $43.26 \%, 33 \%, 44.76 \%$, and $35.44 \%$, respectively, compared with KNN, Rank, Coverage-area, and GPR. The positioning accuracy of the proposed method was improved by $45.72 \%, 55.98 \%, 65.79 \%, 38.26 \%$, respectively, compared to that of KNN, Rank, Coverage-area, and GPR.

Table 5. The statistical results of the positioning errors in Scenarios A and B/m.

\begin{tabular}{ccc}
\hline Method & ME & RMSE \\
\hline KNN & 2.278 & 2.677 \\
Rank & 3.262 & 4.235 \\
Coverage-area & 3.369 & 3.936 \\
GPR & 2.002 & 2.353 \\
Proposed method & 1.236 & 1.519 \\
\hline
\end{tabular}

\section{Conclusions}

In this paper, we proposed a fingerprint positioning algorithm for dual-band Wi-Fi based on the Gaussian process regression and K-nearest neighbor algorithm, which has a better positioning effect than that of the $2.4 \mathrm{GHz}$ fingerprint, $5 \mathrm{GHz}$ fingerprint, and hybrid fingerprint. The proposed algorithm can make full use of the existing location information (2.4 GHz and $5 \mathrm{GHz}$ RSS measurements), obtaining a better positioning accuracy compared with KNN, Rank, Coverage-area, and GPR. The experimental results also show that the proposed method is greatly improved compared with the traditional fingerprint positioning methods.

However, this proposed method needs to determine the basic information of dualband Wi-Fi devices to build the dual-band fingerprint database in advance, which may influence the application of this method.

Author Contributions: Conceptualization, Hongji Cao; Data curation, Jingxue Bi, Hongji Cao, Hongxia Qi, Shenglei Xu, Meng Sun; Formal analysis, Hongji Cao and Jingxue Bi; Funding acquisition, Yunjia Wang; Methodology, Jingxue Bi; Project administration, Yunjia Wang; Software, Jingxue Bi; Supervision, Yunjia Wang; Validation, Jingxue Bi, Hongxia Qi; Visualization, Hongji Cao; Writing-original draft, Hongji Cao. All authors have read and agreed to the published version of the manuscript.

Funding: This research was funded by the National Key Research and Development Program of China [grant number 2016YFB0502102] and National Natural Science Foundation of China [grant number 42001397]. The research was also funded by the State Key Laboratory of Satellite Navigation System and Equipment Technology (CEPNT-2018KF-03), Doctoral Research Fund of Shandong Jianzhu University (XNBS1985), and Key Laboratory of Surveying and Mapping Science and Geospatial Information Technology of Ministry of Natural Resources (2020-3-4).

Institutional Review Board Statement: Not applicable.

Informed Consent Statement: Not applicable.

Data Availability Statement: Not applicable.

Acknowledgments: The authors thank the editors and reviewers of this paper for their comments with which its quality was improved.

Conflicts of Interest: The authors declare no conflict of interest.

\section{References}

1. Ardiansyah, A.; Nugraha, G.D.; Han, H.; Deokjai, C.; Kim, J. A decision tree-based NLOS detection method for the UWB indoor location tracking accuracy improvement. Int. J. Commun. Syst. 2019, 32, e3997.

2. Yu, K.; Wen, K.; Li, Y.; Zhang, S.; Zhang, K. A Novel NLOS Mitigation Algorithm for UWB Localization in Harsh Indoor Environments. IEEE Trans. Veh. Technol. 2019, 68, 686-699. [CrossRef] 
3. Chen, L.; Pei, L.; Kuusniemi, H.; Chen, Y.; Kröger, T.; Chen, R. Bayesian Fusion for Indoor Positioning Using Bluetooth Fingerprints. Wirel. Pers. Commun. 2012, 70, 1735-1745. [CrossRef]

4. Topak, F.; Pekeriçli, M.K.; Tanyer, A.M. Technological Viability Assessment of Bluetooth Low Energy Technology for Indoor Localization. J. Comput. Civ. Eng. 2018, 32, 04018034. [CrossRef]

5. Sun, W.; Xue, M.; Yu, H.; Tang, H.; Lin, A. Augmentation of fingerprints for indoor WiFi localization based on Gaussian process regression. IEEE Trans. Veh. Technol. 2018, 67, 10896-10905. [CrossRef]

6. Oh, J.; Kim, J. Adaptive K-nearest neighbour algorithm for WiFi fingerprint positioning. ICT Express 2018, 4, 91-94. [CrossRef]

7. Zhang, W.; Hua, X.; Yu, K.; Qiu, W.; Zhang, S.; He, X. A novel WiFi indoor positioning strategy based on weighted squared Euclidean distance and local principal gradient direction. Sens. Rev. 2018, 39. [CrossRef]

8. Zhang, Y.; Zhang, S.; Li, R.; Guo, D.; Wei, Y.; Sun, Y. WiFi fingerprint positioning based on clustering in mobile crowdsourcing system. In Proceedings of the 2017 12th International Conference on Computer Science and Education (ICCSE), Houston, TX, USA, 22-25 August 2017; pp. 252-256.

9. Guo, X.; Li, L.; Ansari, N.; Liao, B. Accurate WiFi Localization by Fusing a Group of Fingerprints via a Global Fusion Profile. IEEE Trans. Veh. Technol. 2018, 67, 7314-7325. [CrossRef]

10. Tewolde, G.S.; Kwon, J. Efficient WiFi-Based Indoor Localization Using Particle Swarm Optimization. In Proceedings of the Advances in Swarm Intelligence, Chongqing, China, 12-15 June 2011; pp. 203-211.

11. Xu, H.; Ding, Y.; Li, P.; Wang, R.; Li, Y. An RFID indoor positioning algorithm based on Bayesian probability and K-nearest neighbor. Sensors 2017, 17, 1806. [CrossRef] [PubMed]

12. Xiao, A.; Ruizhi, C.; Deren, L.; Yujin, C.; Dewen, W. An Indoor Positioning System Based on Static Objects in Large Indoor Scenes by Using Smartphone Cameras. Sensors 2018, 18, 2229. [CrossRef] [PubMed]

13. Khyam, M.O.; Noor-A-Rahim, M.; Li, X.; Ritz, C.; Guan, Y.L.; Ge, S.S. Design of Chirp Waveforms for Multiple-Access Ultrasonic Indoor Positioning. IEEE Sens. J. 2018, 18, 6375-6390. [CrossRef]

14. Shi, J. An INS/WiFi Indoor Localization System Based on the Weighted Least Squares. Sensors 2018, 18, 1458. [CrossRef]

15. Li, X.; Zhang, P.; Huang, G.; Zhang, Q.; Zhao, Q. Performance analysis of indoor pseudolite positioning based on the unscented Kalman filter. GPS Solut. 2019, 23, 79. [CrossRef]

16. Ma, Y.; Dou, Z.; Jiang, Q.; Hou, Z. Basmag: An Optimized HMM-Based Localization System Using Backward Sequences Matching Algorithm Exploiting Geomagnetic Information. IEEE Sens. J. 2016, 16, 7472-7482. [CrossRef]

17. Ashraf, I.; Hur, S.; Bin Zikria, Y.; Park, Y. Ensembling Neural Networks for User's Indoor Localization Using Magnetic Field Data from Smartphones. Comput. Mater. Contin. 2021, 68, 2597-2620. [CrossRef]

18. Bi, J.; Wang, Y.; Li, X.; Cao, H.; Qi, H.; Wang, Y. A novel method of adaptive weighted K-nearest neighbor fingerprint indoor positioning considering user's orientation. Int. J. Distrib. Sens. Netw. 2018, 14, 1550147718785885. [CrossRef]

19. Wu, G.; Tseng, P. A Deep Neural Network-Based Indoor Positioning Method using Channel State Information. In Proceedings of the 2018 International Conference on Computing, Networking and Communications (ICNC), Maui, HI, USA, 5-8 March 2018; pp. 290-294.

20. Wang, P.; Luo, Y. Research on WiFi Indoor Location Algorithm Based on RSSI Ranging. In Proceedings of the 20174 th International Conference on Information Science and Control Engineering (ICISCE), Changsha, China, 21-23 July 2017; pp. $1694-1698$.

21. Fang, X.; Jiang, Z.; Nan, L.; Chen, L. Optimal weighted K-nearest neighbour algorithm for wireless sensor network fingerprint localisation in noisy environment. IET Commun. 2018, 12, 1171-1177. [CrossRef]

22. Sharp, I.; Yu, K. Enhanced Least-Squares Positioning Algorithm for Indoor Positioning. IEEE Trans. Mob. Comput. 2013, 12, 1640-1650. [CrossRef]

23. Zhuang, Y.; Syed, Z.; Li, Y.; El-Sheimy, N. Evaluation of Two WiFi Positioning Systems Based on Autonomous Crowdsourcing of Handheld Devices for Indoor Navigation. IEEE Trans. Mob. Comput. 2016, 15, 1982-1995. [CrossRef]

24. Xie, Y.; Wang, Y.; Nallanathan, A.; Wang, L. An Improved K-Nearest-Neighbor Indoor Localization Method Based on Spearman Distance. IEEE Signal Process. Lett. 2016, 23, 351-355. [CrossRef]

25. Bi, J.; Wang, Y.; Li, X.; Qi, H.; Cao, H.; Xu, S. An adaptive weighted KNN positioning method based on omnidirectional fingerprint database and twice affinity propagation clustering. Sensors 2018, 18, 2502. [CrossRef] [PubMed]

26. Beomju, S.; Jung Ho, L.; Taikjin, L.; Hyung Seok, K. Enhanced weighted K-nearest neighbor algorithm for indoor Wi-Fi positioning systems. In Proceedings of the 2012 8th International Conference on Computing Technology and Information Management (NCM and ICNIT), Seoul, Korea, 24-26 April 2012; pp. 574-577.

27. Song, X.; Fan, X.; Xiang, C.; Ye, Q.; Liu, L.; Wang, Z.; He, X.; Yang, N.; Fang, G. A novel convolutional neural network based indoor localization framework with WiFi fingerprinting. IEEE Access 2019, 7, 110698-110709. [CrossRef]

28. Yiu, S.; Yang, K. Gaussian process assisted fingerprinting localization. IEEE Internet Things J. 2015, 3, 683-690. [CrossRef]

29. Youssef, M.; Agrawala, A. The Horus location determination system. Wirel. Netw. 2008, 14, 357-374. [CrossRef]

30. Torres-Sospedra, J.; Moreira, A.; Knauth, S.; Berkvens, R.; Montoliu, R.; Belmonte, O.; Trilles, S.; João Nicolau, M.; Meneses, F.; Costa, A.; et al. A realistic evaluation of indoor positioning systems based on Wi-Fi fingerprinting: The 2015 EvAAL-ETRI competition. J. Ambient. Intell. Smart Environ. 2017, 9, 263-279. [CrossRef]

31. Ali, M.U.; Hur, S.; Park, S.; Park, Y. Harvesting Indoor Positioning Accuracy by Exploring Multiple Features From Received Signal Strength Vector. IEEE Access 2019, 7, 52110-52121. [CrossRef] 
32. Zhang, Y.; Li, D.; Wang, Y. An Indoor Passive Positioning Method Using CSI Fingerprint Based on Adaboost. IEEE Sens. J. 2019, 19, 5792-5800. [CrossRef]

33. Ashraf, I.; Hur, S.; Park, Y. Indoor Positioning on Disparate Commercial Smartphones Using Wi-Fi Access Points Coverage Area. Sensors 2019, 19, 4351. [CrossRef]

34. Lohan, E.S.; Torres-Sospedra, J.; Leppäkoski, H.; Richter, P.; Peng, Z.; Huerta, J. Wi-Fi Crowdsourced Fingerprinting Dataset for Indoor Positioning. Data 2017, 2, 32. [CrossRef]

35. Farshad, A.; Jiwei, L.; Marina, M.K.; Garcia, F.J. A microscopic look at WiFi fingerprinting for indoor mobile phone localization in diverse environments. In Proceedings of the International Conference on Indoor Positioning and Indoor Navigation, Montbeliard, France, 28-31 October 2013; pp. 1-10.

36. Yu, F.; Jiang, M.; Liang, J.; Qin, X.; Hu, M.; Tao, P.; Hu, X. 5G WiFi Signal-Based Indoor Localization System Using Cluster -Nearest Neighbor Algorithm. Int. J. Distrib. Sens. Netw. 2014, 10, 247525. [CrossRef]

37. Karlsson, F.; Karlsson, M.; Bernhardsson, B.; Tufvesson, F.; Persson, M. Sensor fused indoor positioning using dual band WiFi signal measurements. In Proceedings of the 2015 European Control Conference (ECC), Linz, Austria, 15-17 July 2015; pp. 1669-1672.

38. Feng, Y.; Jiang, M.; Jing, L.; Xiao, Q.; Hu, X. Expansion RSS-based Indoor Localization Using 5G WiFi Signal. In Proceedings of the 2014 International Conference on Computational Intelligence and Communication Networks (CICN), Bhopal, India, 14-16 November 2014.

39. Own, C.M.; Hou, J.; Tao, W. Signal Fuse Learning Method with Dual Bands WiFi Signal Measurements in Indoor Positioning. IEEE Access 2019, 7, 131805-131817. [CrossRef] 